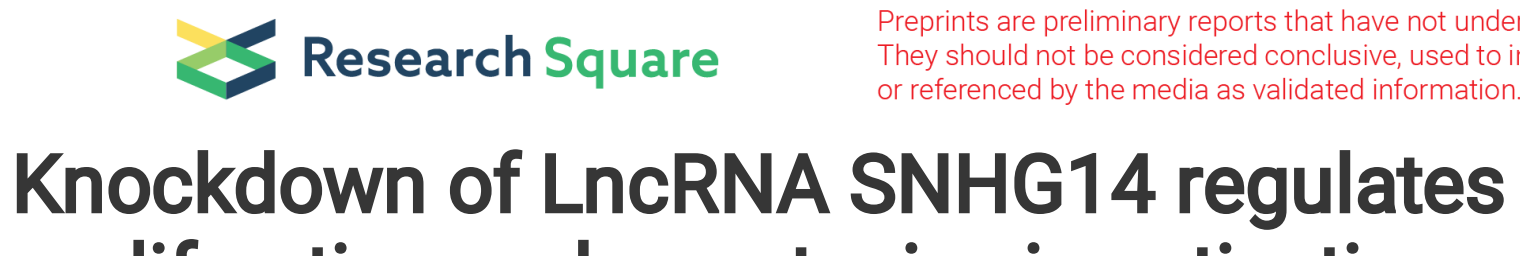 \\ proliferation and apoptosis via activating miR-204- 5p/HMGA1 axis in retinoblastoma
}

\section{Xuedong Chen}

The First Affiliated Hospital of Harbin Medical University

\section{Xianling Tang}

The First Affiliated Hospital of Harbin Medical University

\section{Shiyong Zhao}

The Second Affiliated Hospital of Harbin Medical University

\section{Yongbin Yu}

The First Affiliated Hospital of Harbin Medical University

\section{Qingjun Li}

The First Affiliated Hospital of Harbin Medical University

\section{Caicai Xu}

The First Affiliated Hospital of Harbin Medical University

Hongyan Ge ( $\nabla$ exb0zzj@163.com )

The First Affiliated Hospital of Harbin Medical University https://orcid.org/0000-0001-5566-4056

\section{Primary research}

Keywords: SNHG14, miR-204-5p, HMGA1, retinoblastoma, proliferation, apoptosis

Posted Date: February 20th, 2020

DOl: https://doi.org/10.21203/rs.2.24066/v1

License: (9) (1) This work is licensed under a Creative Commons Attribution 4.0 International License. Read Full License 


\section{Abstract}

Background: Retinoblastoma (RB) is an aggressive intraocular malignancy of infant and childhood, which seriously endangers the vision and life of children. Long non-coding RNA Small Nucleolar RNA Host Gene 14 (IncRNA SNHG14) as a novel oncogene is involved in the control of cancer cell progression. However, the effects and molecular mechanism of SNHG14 on retinoblastoma remain confusing.

Methods: Levels of SNHG14, high mobility group protein A1 (HMGA1) mRNA and microRNA (miR)-204-5p were detected by quantitative real-time polymerase chain reaction. Cell proliferation and apoptosis were measured by CKK-8 assay or flow cytometry, respectively. Western blot was used to detect HMGA1 and apoptosis-related protein. The interaction between miR-204-5p and SNHG14 or HMGA1 was explored by luciferase reporter assay. Murine xenograft model was established using Y79 cells stably transfected with shSNHG14.

Results: SNHG14 and HMGA1 were up-regulated in retinoblastoma tissues and cell lines, and knockdown of SNHG14 or HMGA1 suppressed cell proliferation and induced cell apoptosis in retinoblastoma. MiR-204-5p was confirmed to directly bind to SNHG14 and HMGA1, SNHG14 positively regulated HMGA1 expression via miR-204-5p. Importantly, the anti-tumor effects mediated by SNHG14 knockdown could be reversed by miR204-5p inhibition or HMGA1 overexpression in retinoblastoma cells. Furthermore, xenograft model showed SNHG14 silence impeded tumor growth in vivo.

Conclusion: Knockdown of SNHG14 suppressed retinoblastoma progression by regulating miR-2045p/HMGA1 axis, revealing a potential target to develop appropriate treatment strategies for retinoblastoma.

\section{Background}

Retinoblastoma $(\mathrm{Rb})$ is the most common primary intraocular malignancy in infants and children, which seriously endangers the vision and life of children [1]. At present, the survival rate of children with retinoblastoma in China is still low, and the enucleation rate of the eyeball is still higher. The main reason is that most children with retinoblastoma have entered the middle and late stage of treatment, and have to survive through eyeball enucleation or even orbital enucleation [2, 3]. Therefore, better understanding on the mechanisms of the occurrence and development of RB is extremely important to improve the treatment strategies and quality of life of children with retinoblastoma.

Long non-coding RNAs (IncRNAs), longer than 200 nucleotides, are a class of RNA molecules without the ability to encode proteins [4]. Recent studies have shown that IncRNAs are involved in many important regulatory processes, such as X chromosome silencing, genomic imprinting, chromatin modification, transcriptional activation, transcriptional interference and nuclear transport, etc. [5]. In addition, findings from recent studies reveal the potential effects in both tumor-suppressing pathways and oncogenic of IncRNA. An oncogenic IncRNAs may cause uncontrolled progression of tumor cells via regulating epigenetics and multiple signaling pathways, such as cell proliferation, apoptosis, metastasis and so on [6, 7]. LncRNA Small Nucleolar RNA Host Gene 14 (SNHG14), also named antisense of ubiquitin protein ligase E3A (UBE3A-ATS), is a $19.2 \mathrm{~kb}$ transcript and mapped on chromosome 15q11.2. SNHG14 can overlap with the entire UBE3A gene and promoter in antisense direction, thus silencing the expression of UBE3A to result in neurogenetic disorders [8, 
9]. Recently emerging evidence has reported that SNHG14 functions as an oncogene in several malignancies to contribute to the evolution of cancers, including glioma [10], gastric cancer [11], colorectal cancer [12], lung cancer[13] and so on. However, the function and molecular mechanism of SNHG14 on retinoblastoma remain confusing.

MicroRNAs (miRNAs) are small non-coding RNAs, which are implicate in multifarious biological process though targeting sequence of cellular and molecular pathways [14]. Dyregulation of miRNAs is revealed to be involved in various types of diseases cancerous condition, including retinoblastoma [15]. MiRNAs may act as oncogenes or tumor suppressor genes depending on the target genes. Recently, miR-204-5p has been identified to function as a tumor suppressor to inhibit cancer cell growth $[16,17]$. While the role of miR-204-5p in retinoblastoma is still overlooked. High mobility group A1 (HMGA1) is located on chromosome 6p21 in humans. HMGA1 is lower expression in adult tissues, but is enriched during embryogenesis, indicating their important role in development [18]. Previous findings indicated that HMGA1 overexpression disturbs retinoblastoma protein (RB)-mediated cell arrest; besides, the interaction between HMGA1 and RB is required for adipocyte differentiation, suggesting a negative control of retinoblastoma by HMGA1 [19, 20].

In the present study, we aimed to investigate the function of SNHG14 on retinoblastoma proliferation and apoptosis, explored the molecular mechanism of the action of SNHG14 on retinoblastoma, as well as the relationship among SNHG14, HMGA1 and miR-204-5p in the progression of retinoblastoma.

\section{Materials And Methods}

\section{Patients and specimens}

A total of 30 primary retinoblastoma tissues and 15 normal retina samples from ruptured globes were obtained, who received surgery treatment, were obtained from The First Affiliated Hospital of Harbin Medical University between September 2017 and January 2018. All specimens were confirmed by two independent pathologists and did not receive radiotherapy or chemotherapy. Specimens were immediately stored at $-80^{\circ} \mathrm{C}$ until experiment.

\section{Cell culture and transfection}

ARPE-19, WERI-RB1, and Y79 cell lines were purchased from American Type Culture Collection (ATCC). HXORB44 was obtained from Xi'an Jiaotong University, and SO-Rb50 was provided from the Department of Pathology of the Sun Yat-sen University. All cells were cultured in RPMI-1640 medium (Gibco, Carlsbad, CA, USA) containing $10 \%$ fetal bovine serum (FBS) (Gibco) at $37^{\circ} \mathrm{C}$ with $5 \% \mathrm{CO}_{2}$.

The miR-204-5p mimic (miR-204-5p), mimic negative control (miR-NC), miR-204-5p inhibitor (anti-miR-204-5p), inhibitor negative control (anti-miR-NC), short hairpin RNA (sh-RNA) against SNHG14 (sh-SNHG14), sh-RNA against HMGA1 (sh-HMGA1) or sh-RNA negative control (sh-NC) were purchased from Genepharma (Shanghai, China). The pcDNA3.1-HMGA1 (HMGA1) or pcDNA3.1-control (pcDNA) was obtained from Invitrogen (Carlsbad, CA, USA). They were transfected into HXO-Rb44 and Y79 cell lines using Lipofectamine 2000, respectively, and cells were harvested 48 hours after transfection for further analysis. 


\section{Quantitative real-time polymerase chain reaction (qRT-PCR)}

Total RNA was isolated from retinoblastoma tissues and cells using TRIzol reagent (Invitrogen). Then cDNA was synthesized with reverse transcriptase and obtained CDNA was subjected to quantitative real-time polymerase chain reaction (qRT-PCR) with SYBR Green master kit (Takara, Dalian, China) following the instruction of manufacturer. GAPDH or U6 was as an endogenous control and fold changes were calculated using the $2^{-\triangle \triangle C t}$ method. All special primers were listed as follows, SNHG14 forward, $5^{\prime}$ GGGTGTTTACGTAGACCAGAACC-3', reverse 5'-TCACTGGGTGCCATCGTAAGAA-3'; HMGA1 forward 5'CTTCCAAAAGCCTTCTGCCTTAG-3'; reverse 5'-CCCCGAGGTCTCTTAGG- TGTTGG-3'; GAPDH forward 5'GGATGCAGCCTCACAGTCCTTAC-3', reverse 5'-GGCCTCAGGGTCCAAAGACA-3'; miR-204-5p 5'UUCCCUUUGUCAUCC- UAUGCCUG-3'; U6 forward 5'-CGAGCACAGAATCGCTTCA-3'; reverse 5'CTCGCTTCGGCAGCACATAT-3'.

\section{Cell proliferation and apoptosis}

Cell Counting Kit-8 (CCK8) was used to measure the proliferation of retinoblastoma cells. All cells were seeded into 96 -well plates at a density of $3000 /$ well after transfection and maintained at $37^{\circ} \mathrm{C}$ with $5 \% \mathrm{CO}_{2}$ for $24 \mathrm{~h}$. Then $10 \mu \mathrm{L}$ CCK-8 reagents (Sigma, St. Louis, MO, USA) were added to the each plate and incubated for $2 \mathrm{~h}$. Finally, the absorbance value of each test well was measured at $450 \mathrm{~nm}$ by a microplate reader.

Apoptosis rates were detected by flow cytometry. After transfection, cells were incubated with an Annexin VFITC/PI apoptosis detection kit (Solarbio, Beijing, China) according to the protocol of manufacturer. Finally, apoptotic cells were detected using ModFit LT software.

\section{Western blot}

Transfected or untransfected cells were lysed within RIPA buffer (Beyotime, Shanghai, China). A total of $50 \mu \mathrm{g}$ extracted protein were loaded onto 8\% SDS-PAGE microgels, transferred to PVDF membranes (Millipore), and then blocked with $5 \%$ non-fat milk for $1 \mathrm{~h}$ at room temperature. Subsequently, the membranes were incubated with primary antibodies against B-cell lymphoma-2 (Bcl-2) (1:1000, ab692, Abcam, Cambridge, MA, USA), BCL2-associated X protein (Bax) (1:1000, ab32503, Abcam), cleaved-casp-3 (1:5000, ab2302, Abcam), HMGA1 (1:10000, ab129153, Abcam) overnight at $4^{\circ} \mathrm{C}$, and after washed with TBST for four times, the membranes were interacted with HRP-conjugated secondary antibody (1:5000, Sangon, Shanghai, China) for 2h. GAPDH was used as normalized endogenous control. The signals were visualized with ECL Substrates (Millipore, Billerica, MA, USA).

\section{Luciferase reporter assay}

The SNHG14 or HMGA1 3'UTR fragments containing wild-type (wt) or mutant (mut) putative target sites of miR-204-5p were cloned into pmirGLO vectors (Promega, Madison, WI, USA) to generate pmirGLO-WT-SNHG14 (WT-SNHG14), pmirGLO-MUT-SNHG14 (MUT-SNHG14), pmirGLO-HMGA1 3'UTR-WT (HMGA1 3'UTR-WT) or MUT (HMGA1 3'UTR-MUT), respectively. Subsequently, $40 \mathrm{nM}$ of miR-204-5p mimic or miR-NC and $200 \mathrm{ng}$ of constructed luciferase reporter plasmids were co-transfected into HXO-Rb44 and Y79 cells for 48h using 
Lipofectamine 2000 (Invitrogen). Relative luciferase activity was measured using dual luciferase assay kit (Promega) and normalizing to renilla luciferase activity.

\section{Murine xenograft assay}

BALB/c nude mice randomly divided into two groups (Male, 6 weeks old, $n=8$ for each group) were purchased from Vital River Laboratories Company (Beijing, China). Y79 cells $\left(1 \times 10^{6}\right)$ transfected with the lentivirus-shSNHG14 (sh-SNHG14) or negative control (sh-NC) were subcutaneously injected into the right-side flanks of of BALB/c nude mice. Tumor size was measured regularly every three days. At day 27 , all mice were sacrificed, and the tumors samples were weighed and used for further analyzed.

\section{Statistical analysis}

All statistical analyses of data were conducted by GraphPad Prism 7 (GraphPad Inc., San Diego, CA, USA). All data are expressed as mean \pm SD. Student's $t$ test and one-way analysis of variance (ANOVA) were used to analyze the difference between groups. $P<0.05$ was considered statistically significant.

\section{Results}

\section{The expression of SNHG14 on retinoblastoma tissues and cells}

The expression of SNHG14 in human retinoblastomas tissues and cells line was analyzed though qRT-PCR, and the data showed the level of SNHG14 was significantly increased in retinoblastoma tissues compared to in normal retina samples (Fig 1A). Similar, a notably up-regulation of SNHG14 expression in retinoblastoma cell lines (including WERI-RB1, SO-Rb50, HXO-Rb44 and Y79) compared to the normal retinoblastoma epithelial cell line APRE-19 was observed (Fig 1B). Thus, SNHG14 increase might be associated the development and progression.

\section{Knockdown of SNHG14 inhibits proliferation and promotes apoptosis in retinoblastoma cells}

To verify the effects of SNHG14 on retinoblastoma cells, HXO-Rb44 and Y79 cells were transfected with shSNHG14 or sh-NC, and a down-regulation of SNHG14 expression was observed in HXO-Rb44 and Y79 cells (Fig 2A). Subsequently, the effects of SNHG14 on cell proliferation and apoptosis were evaluated. CCK-8 assay showed knockdown of SNHG14 inhibited the proliferation of HXO-Rb44 and Y79 cells (Fig 2B, C). Meanwhile, the abrogation of SNHG14 induced apoptosis of HXO-Rb44 and Y79 cells (Fig 2D); besides that, SNHG14 silence reduced the level of Bcl-2, while elevated the level of cleaved-casp-3 and Bax in HXO-Rb44 and $Y 79$ cells (Fig 2E), further suggesting SNHG14 silence promoted cell apoptosis. Taken together, Knockdown of SNHG14 inhibited the progression of retinoblastoma.

\section{SNHG14 targeted miR-204-5p to promote retinoblastoma tumor progression}

To investigate the underlying molecular mechanism of action of SNHG14 in retinoblastoma progression, the potential miRNA targets of SNHG14 were explored by bioinformatics analysis via TargetScan software, and SNHG14 was predicted to bind to miR-204-5p with a putative binding site (Fig 3A). MiR-204-5p was found to be significantly decreased in retinoblastoma tissues and cells compared to the normal control (Fig 3B, D), and 
was negatively correlated with SNHG14 expression in retinoblastoma tissues $(r=-0.401 ; p=0.028)$ (Fig 3C).

Subsequently, the dual luciferase reporter analysis showed that luciferase activity was conspicuously inhibited in HXO-Rb44 and Y79 cells transfected with WT-SNHG14 and miR-204-5p compared with the control, while there was no change in MUT-SNHG14 group (Fig 3E, F), indicating the direct interaction of SNHG14 and miR204-5p. Additionally, we also found knockdown of SNHG14 significantly up-regulated the expression level of miR-204-5p (Fig 3G). Thus, we confirmed that SNHG14 targetedly suppressed miR-204-5p expression.

Base on the relationship between miR-204-5p and SNHG14, we detected whether the activity of SNHG14 on retinoblastoma cell was mediated by miR-204-5p. First, HXO-Rb44 and Y79 cells were transfected with antimiR-204-5p or anti-NC, and anti-miR-204-5p transfection significantly reduced the level of miR-204-5p in HXORb44 and Y79 cells compared with anti-NC transfection (Fig. 3H). Next, sh-NC, sh-SNHG14, sh-SNHG14 + antiNC or sh-SNHG14 + anti-miR-204-5p was transfected into HXO-Rb44 and Y79 cells to conducted rescue assay. We discovered that miR-204-5p inhibition markedly reversed SNHG14 knockdown-mediated proliferation inhibition (Fig. 3l, J) and apoptosis promotion (Fig. 3K-M) in HXO-Rb44 and Y79 cells. These data indicated knockdown of SNHG14 regulated retinoblastoma progression by binding to miR-204-5p.

\section{The expression and effects of HMGA1 on retinoblastoma}

The expression level of HMGA1 were measured, and HMGA1 was significantly up-regulated in retinoblastoma tissues at mRNA and protein levels (Fig 4A, C); besides, HMGA1 mRNA expression was positively associated with SNHG14 ( $r=0.411, p=0.024)$ (Fig 4B). Also, relative to the normal APRE-19 cells, HMGA1 was elevated in HXO-Rb44 and Y79 cells (Fig 4D, E). Subsequently, HXO-Rb44 and Y79 cells were transfected with the sh-NC and sh-HMGA1 to explore the role of HMGA1 in retinoblastoma, and HMGA1 expression was significantly declined by sh-HMGA1 in HXO-Rb44 and Y79 cells (Fig 4F, G). After that, cell proliferative vitality was detected using CCK8 assays, and results illustrated HMGA1 knockdown inhibited Y79 and HXO-Rb44 cell proliferation (Fig $4 \mathrm{H}, \mathrm{I})$. Then flow cytometry and western bolt analysis indicated down-regulation of HMGA1 induced apoptosis of HXO-Rb44 and Y79 cells (Fig 4J, K). These results suggested HMGA1 silencing inhibited cell progression in retinoblastoma.

\section{HMGA1 is a target of miR-204-5p}

Considering the aberrantly expression of miR-204-5p and HMGA1 in retinoblastoma cells, we further studied the relationship between miR-204-5p and HMGA1. Though an online software TargetScan, we found HMGA1 might be a target of miR-204-5p (Fig 5A). Then a decline of luciferase activity in HXO-Rb44 and Y79 cells cotransfected with HMGA1 3'UTR-WT and miR-204-5p confirmed the direct interaction of miR-204-5p and HMGA1 (Fig 5B, C). Importantly, the expression of HMGA1, both mRNA and protein, was inhibited by miR-204$5 p$ overexpression, but was elevated by miR-204-5p down-regulation in HXO-Rb44 and Y79 cells (Fig 5D-G). Thus, we verified that miR-204-5p targetedly suppressed HMGA1 expression.

\section{SNHG14 inhibits proliferation and induces apoptosis by regulating the expression of HMGA1 via miR-204-5p in retinoblastoma cells}

Given SNHG14 sponged miR-204-5p, and miR-204-5p targeted HMGA1, we investigated whether SNHG14 could regulate HMGA1 through miR-204-5p. The expression of HMGA1 was detected in HXO-Rb44 and Y79 
cells transfected with sh-NC, sh-SNHG14, sh-SNHG14 + anti-NC or sh-SNHG14 + anti-miR-204-5p. Results exhibited that the level of HMGA1 was suppressed by SNHG14 knockdown, while was rescued by miR-204-5p inhibition (Fig 6A-B), suggesting SNHG14 regulated HMGA1 expression via miR-204-5p. Subsequently, HXORb44 and Y79 cells were transfected with pcDNA or HMGA1, and HMGA1 expression was significantly increased in the HMGA1 transfected cells (Fig 6C), showing the successful transfection (Fig 6D). Next, sh-NC, sh-SNHG14, sh-SNHG14 + pcDNA, or sh-SNHG14 + HMGA1 was transfected into HXO-Rb44 and Y79 cells to performed rescues assay. We discovered that HMGA1 overexpression abated the inhibition on cell proliferation (Fig 6E, F) and the promotion on apoptosis (Fig 6G-I) mediated by SNHG14 knockdown in HXO-Rb44 and Y79 cells. Altogether, SNHG14 regulate retinoblastoma cell tumorigenesis by HMGA1.

\section{SNHG14 knockdown inhibits retinoblastoma tumor growth in vivo}

The role of SNHG14 in tumor growth in vivo was explored. As presented in Fig. 7A, B, SNHG14 knockdown impeded tumor growth, reflected by the decrease of tumor volume (Fig. 7A) and weight (Fig. 7B) in sh-SNHG14 group relative to the sh-NC group. Subsequently, molecular analysis in tumor masses showed SNHG14 silence reduced the levels of SNHG14 (Fig. 7C) and HMGCR (Fig. 7C, D), but elevated the level of miR-204-5p (Fig. 7C). Thus, we concluded that SNHG14 knockdown inhibits retinoblastoma tumor growth in vivo by regulating HMGCR and miR-204-5p.

\section{Discussion}

Retinoblastoma is the most common malignant intraocular tumor in children with an estimated incidence of $1 / 20,000$ live births [21]. In recent year, the treatment of retinoblastoma has significantly improved. There is a growth trend to use conservative treatment to preserve the eyeballs as well as vision with minimum morbidity worldwide; besides that, the use of chemotherapy and radiotherapy to the eye has also shown promising results and are beneficial in retinoblastoma patients $[22,23]$. Nevertheless, challenges remain enormous, especially in the developing country.

Up to date, increasing number of IncRNA have been reported to be associated with the progression of retinoblastoma. For example, AFAP1-AS1 served as an independent unfavorable prognostic factor in retinoblastoma patients, and promoted malignant phenotypes of retinoblastoma cell to contributed the progression of retinoblastoma [24]. LncRNA-H19 enhanced cell viability, migration and invasion but suppressed apoptosis in retinoblastoma by absorbing miR-143 through RUNX2 [25]. MALAT1 promoted retinoblastoma cell autophagy via miR-124-mediated stx17 regulation [26]. Thus, IncRNAs may be potential diagnostic and therapeutic target for retinoblastoma. SNHG14 is a well-recognized oncogene, and has been shown to regulate drug resistance, proliferation, apoptosis and metastasis in numerous cancers, thus affecting survival and prognosis of tumor patients $[10,13]$. However, few studies revealed the role of SNHG14 in retinoblastoma. In the present study, SNHG14 was elevated in retinoblastoma tissues and cells, and SNHG14 knockdown inhibited retinoblastoma cell proliferation and induced apoptosis in vitro. Importantly, murine xenograft model analysis showed SNHG14 silence also hindered tumor growth in vivo. Thus, targeting SNHG14 may be a valuable treatment strategy for retinoblastoma. 
MiR-204-5p has been reported to be down-regulated in several types of cancers such as breast cancer, glioma and prostate cancer, and miR-204-5p inhibition contributes to the progression of cancer cell malignant properties [27-29]. In this study, miR-204-5p was confirmed to be target of SNHG14. MiR-204-5p was decreased in retinoblastoma, and was negatively regulated by SNHG14. Meanwhile, miR-204-5p inhibition was found to reverse the inhibitory effects of SNHG14 knockdown in the progression of retinoblastoma.

HMGA1 has been discovered to richly express in all human neoplastic tissues, including gastric, prostate, colon, testis, breast, lung, ovary, thyroid, pancreas carcinomas and also in some forms of leukemia [30], and highly expression of HMGA1 was related to the development and poor prognosis of cancers [31, 32]. Additionally, it has been revealed that overexpression of HMGA1 is associated with the tumorigenesis and progression of retinoblastomas [33]. In our study, further study was performed to explore the molecular mechanism of miR-204-5p, thus, downstream signal path is predicted. Then we confirmed HMGA1 was a target of miR-204-5p and miR-204-5p targetedly suppressed HMGA1 expression. HMGA1 was elevated in retinoblastoma and silencing of HMGA1 inhibited cell progression in retinoblastoma. In addition, SNHG14 could positively regulate HMGA1 via miR-204-5p, and HMGA1 overexpression attenuated the action of SNHG14 knockdown on retinoblastoma cell proliferation and apoptosis.

\section{Conclusion}

Taken together, our study demonstrated SNHG14 function as an oncogene to promote cell proliferation and induce apoptosis in retinoblastoma through miR-204-5p mediated HMGA1 regulation, providing a novel insight into the occurrence and progression of retinoblastoma, and a prospective therapy strategy for the retinoblastoma treatment.

\section{Abbreviations}

RB: Retinoblastoma; HMGA1: high mobility group protein A1; IncRNAs: Long non-coding RNAs; SNHG14: Small Nucleolar RNA Host Gene 14; miRNAs: MicroRNAs; ATCC: American Type Culture Collection; miR-NC: mimic negative control; pcDNA: pcDNA3.1-control; qRT-PCR: Quantitative real-time polymerase chain reaction; CCK8: Cell Counting Kit-8;

\section{Declarations}

\section{Ethics approval and consent to participate}

The study was approved by the Ethics Committee of The First Affiliated Hospital of Harbin Medical University and written informed consents were collected from all patients and hospitals.

The animal experiment was permitted by the Animal Research Committee of The First Affiliated Hospital of Harbin Medical

University and performed in accordance with the guidelines of the National Animal Care and Ethics Institution.

\section{Consent for publication}


Not applicable

\section{Availability of data and materials}

The data sets used and/or analyzed during the current study are available from the corresponding author on reasonable request.

\section{Competing interests}

The authors declare that they have no conflicts of interests.

\section{Funding}

This study was supported by grant from Heilongjiang Postdoctoral Scientific Research Developmental Fund (No. LBH-Q17093).

\section{Authors' contributions}

Xuedong Chen and Xianling Tang participated in the conception and design of the study. Shiyong Zhao and Yongbin Yu performed the analysis and interpretation of data. Qingjun Li contributed to drafting the manuscript. Caicai Xu and Hongyan Ge reviewed and approved the final submitted manuscript.

\section{Acknowledgment}

Thanks for all participants involved in this study.

\section{References}

1. Abramson DH. Retinoblastoma in the 20th century: past success and future challenges the Weisenfeld lecture. Invest Ophthalmol Vis Sci. 2005, 46(8):2683-91.

2. Chantada GL, Dunkel IJ, Antoneli CB, de Davila MT, Arias V, Beaverson K, Fandino AC, Chojniak M, Abramson DH. Risk factors for extraocular relapse following enucleation after failure of chemoreduction in retinoblastoma. Pediatr Blood Cancer. 2007, 49(3):256-60.

3. Mouratova T. Eye cancer in adults in Uzbekistan, 1978-1998. Bull Soc Belge Ophtalmol. 2004(294):25-34.

4. Ouyang J, Hu J, Chen JL. IncRNAs regulate the innate immune response to viral infection. Wiley Interdiscip Rev RNA. 2016, 7(1):129-43.

5. Jarroux J, Morillon A, Pinskaya M. History, Discovery, and Classification of IncRNAs. Adv Exp Med Biol. 2017, 1008:1-46.

6. Bhan A, Soleimani M, Mandal SS. Long Noncoding RNA and Cancer: A New Paradigm. Cancer Res. 2017, 77(15):3965-81.

7. Huarte M. The emerging role of IncRNAs in cancer. Nat Med. 2015, 21(11):1253-61.

8. Sadikovic B, Fernandes P, Zhang VW, Ward PA, Miloslavskaya I, Rhead W, Rosenbaum R, Gin R, Roa B, Fang P. Mutation Update for UBE 3 A Variants in Angelman Syndrome. Human mutation. 2014, 35(12):1407-17. 
9. Qi X, Shao M, Sun H, Shen Y, Meng D, Huo W. Long non-coding RNA SNHG14 promotes microglia activation by regulating miR-145-5p/PLA2G4A in cerebral infarction. Neuroscience. 2017, 348:98-106.

10. Wang Q, Teng Y, Wang R, Deng D, You Y, Peng Y, Shao N, Zhi F. The long non-coding RNA SNHG14 inhibits cell proliferation and invasion and promotes apoptosis by sponging miR-92a-3p in glioma. Oncotarget. 2018, 9(15):12112-24.

11. Liu Z, Yan Y, Cao S, Chen Y. Long non-coding RNA SNHG14 contributes to gastric cancer development through targeting miR-145/SOX9 axis. J Cell Biochem. 2018, 119(8):6905-13.

12. Ye T, Zhang N, Wu W, Yang B, Wang J, Huang W, Tang D. SNHG14 promotes the tumorigenesis and metastasis of colorectal cancer through miR-32-5p/SKIL axis. In Vitro Cell Dev Biol Anim. 2019, 55(10):812-20.

13. Wu K, Li JC, Qi Y, Zhang C, Zhu D, Liu D, Zhao S. SNHG14 confers gefitinib resistance in non-small cell lung cancer by up-regulating ABCB1 via sponging miR-206-3p. Biomed Pharmacother. 2019, 116:108995.

14. Gholamin S, Mirzaei H, Razavi SM, Hassanian SM, Saadatpour L, Masoudifar A, ShahidSales S, Avan A. GD2-targeted immunotherapy and potential value of circulating microRNAs in neuroblastoma. J Cell Physiol. 2018, 233(2):866-79.

15. Golabchi K, Soleimani-Jelodar R, Aghadoost N, Momeni F, Moridikia A, Nahand JS, Masoudifar A, Razmjoo H, Mirzaei H. MicroRNAs in retinoblastoma: Potential diagnostic and therapeutic biomarkers. $J$ Cell Physiol. 2018, 233(4):3016-23.

16. Luan W, Qian Y, Ni X, Bu X, Xia Y, Wang J, Ruan H, Ma S, Xu B. miR-204-5p acts as a tumor suppressor by targeting matrix metalloproteinases- 9 and B-cell lymphoma-2 in malignant melanoma. Onco Targets Ther. 2017, 10:1237-46.

17. Palkina N, Komina A, Aksenenko M, Moshev A, Savchenko A, Ruksha T. miR-204-5p and miR-3065-5p exert antitumor effects on melanoma cells. Oncol Lett. 2018, 15(6):8269-80.

18. Fusco A, Fedele M. Roles of HMGA proteins in cancer. Nat Rev Cancer. 2007, 7(12):899-910.

19. Ueda Y, Watanabe S, Tei S, Saitoh N, Kuratsu J, Nakao M. High mobility group protein HMGA1 inhibits retinoblastoma protein-mediated cellular G0 arrest. Cancer Sci. 2007, 98(12):1893-901.

20. Esposito F, Pierantoni GM, Battista S, Melillo RM, Scala S, Chieffi P, Fedele M, Fusco A. Interaction between HMGA1 and retinoblastoma protein is required for adipocyte differentiation. J Biol Chem. 2009, 284(38):25993-6004.

21. Cassoux N, Lumbroso L, Levy-Gabriel C, Aerts I, Doz F, Desjardins L. Retinoblastoma: Update on Current Management. Asia Pac J Ophthalmol (Phila). 2017, 6(3):290-5.

22. Chawla B, Singh R. Recent advances and challenges in the management of retinoblastoma. Indian J Ophthalmol. 2017, 65(2):133-9.

23. Mendoza PR, Grossniklaus HE. The Biology of Retinoblastoma. Prog Mol Biol Transl Sci. 2015, 134:50316.

24. Hao F, Mou Y, Zhang L, Wang S, Yang Y. LncRNA AFAP1-AS1 is a prognostic biomarker and serves as oncogenic role in retinoblastoma. Bioscience reports. 2018, 38(3):BSR20180384.

25. Qi D, Wang M, Yu F. Knockdown of IncRNA-H19 inhibits cell viability, migration and invasion while promotes apoptosis via microRNA-143/RUNX2 axis in retinoblastoma. Biomedicine \& Pharmacotherapy. 
2019, 109:798-805.

26. Huang J, Yang Y, Fang F, Liu K. MALAT1 modulates the autophagy of retinoblastoma cell through miR124-mediated stx17 regulation. J Cell Biochem 2018, 119(5):3853-63.

27. Xia ZQ, Liu F, Zhang J, Liu L. Decreased Expression of MiRNA-204-5p Contributes to Glioma Progression and Promotes Glioma Cell Growth, Migration and Invasion. PloS one. 2015, 10(7):e0132399.

28. Liu YC, Liu JF, Tai TF, Chou KY, Chen HE, Huang TS. Tumor suppressor miRNA-204-5p promotes apoptosis by targeting BCL2 in prostate cancer cells. Asian J Surg. 2017, 40(5):396-406.

29. Hong BS, Ryu HS, Kim N, Kim J, Lee E, Moon H, Kim KH, Jin MS, Kwon NH, Kim S, et al. Tumor Suppressor miRNA-204-5p Regulates Growth, Metastasis, and Immune Microenvironment Remodeling in Breast Cancer. Cancer Res. 2019, 79(7):1520-34.

30. De Martino M, Forzati F, Arra C, Fusco A, Esposito F. HMGA1-pseudogenes and cancer. Oncotarget. 2016, 7(19):28724-35.

31. Cleynen I, Van de Ven WJ. The HMGA proteins: a myriad of functions (Review). Int J Oncol. 2008, 32(2):289-305.

32. Fedele M, Fusco A. HMGA and cancer. Biochim Biophys Acta. 2010, 1799(1-2):48-54.

33. Mu G, Liu H, Zhou F, Xu X, Jiang H, Wang Y, Qu Y. Correlation of overexpression of HMGA1 and HMGA2 with poor tumor differentiation, invasion, and proliferation associated with let-7 down-regulation in retinoblastomas. Hum Pathol. 2010, 41(4):493-502.

\section{Figures}
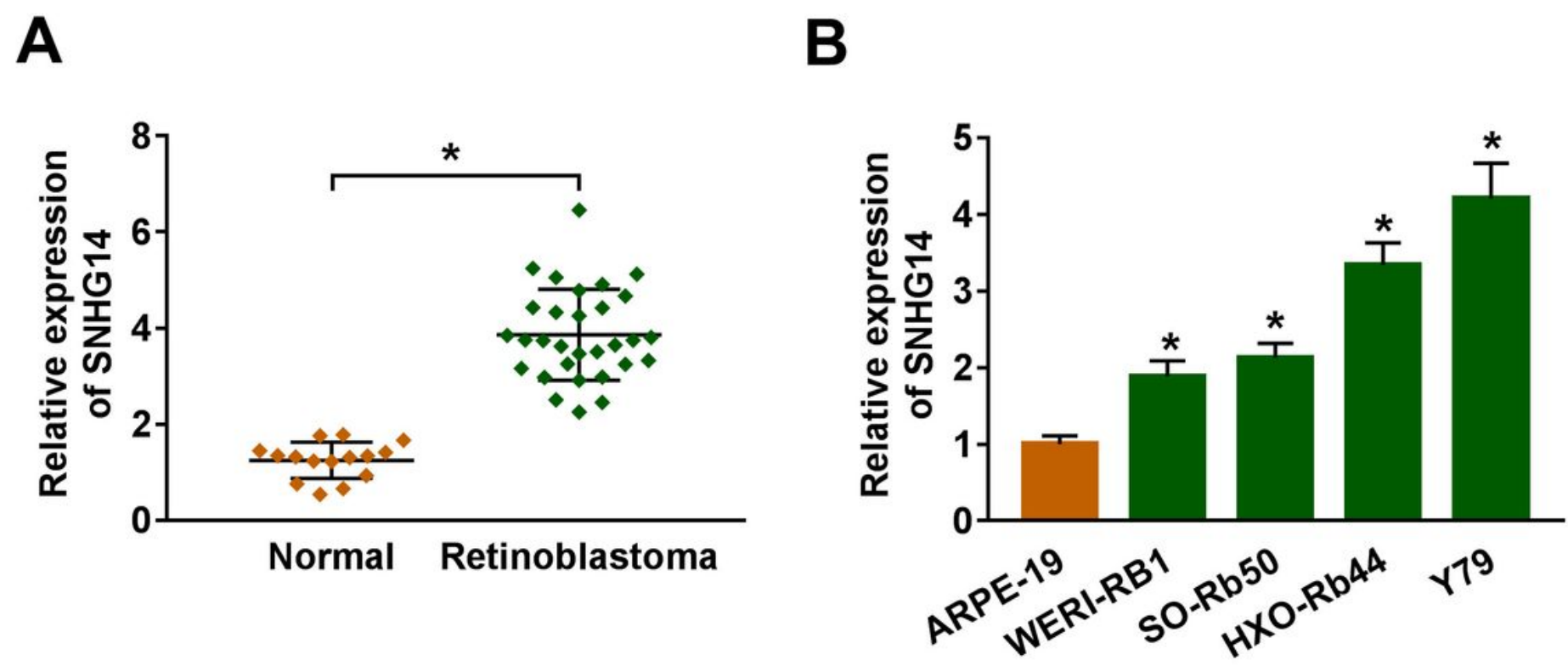

Figure 1

The expression of SNHG14 on retinoblastoma tissues and cells. Levels detection of SNHG14 in retinoblastoma tissues and normal retina samples $(A)$, as well as human retinoblastoma cell lines (WERI-RB1, 
SO-Rb50, HXO-RB44, and Y79) and normal cell line ARPE-19 (B) using qRT-PCR. *P<0.05.

A

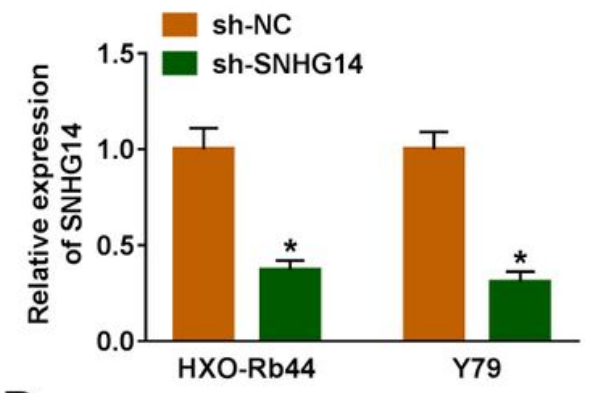

D

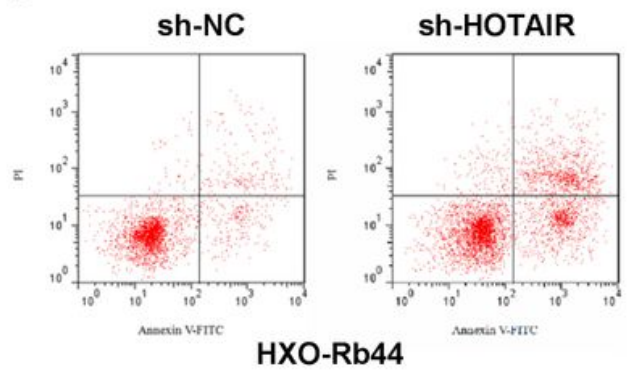

$\mathbf{E}$

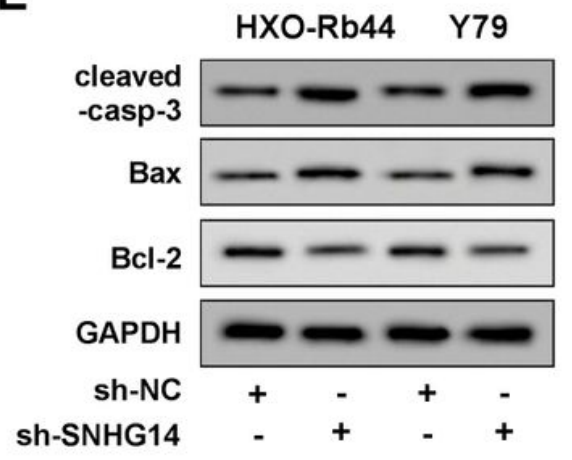

B
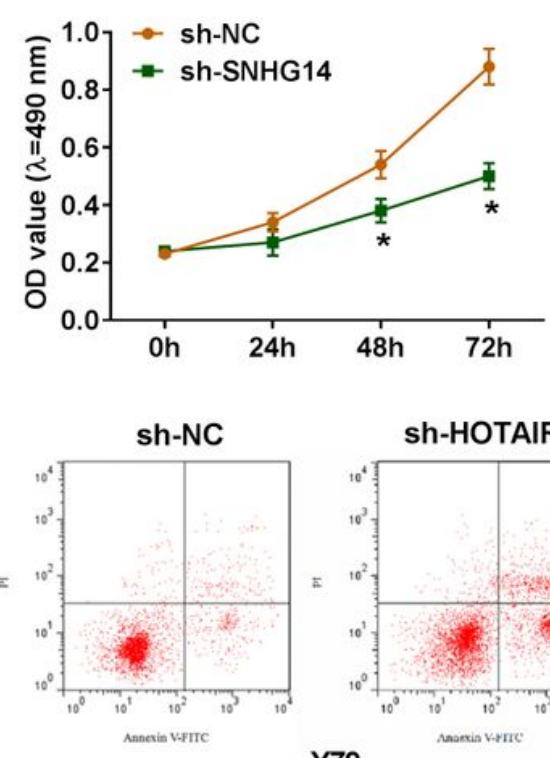

Y79

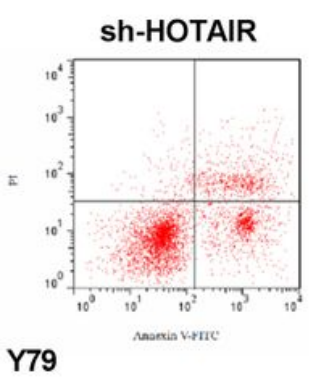

HXO-Rb44

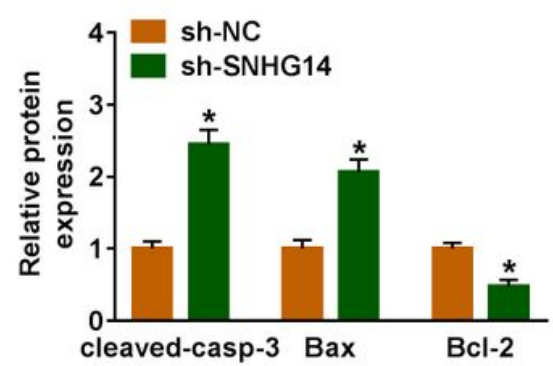

C
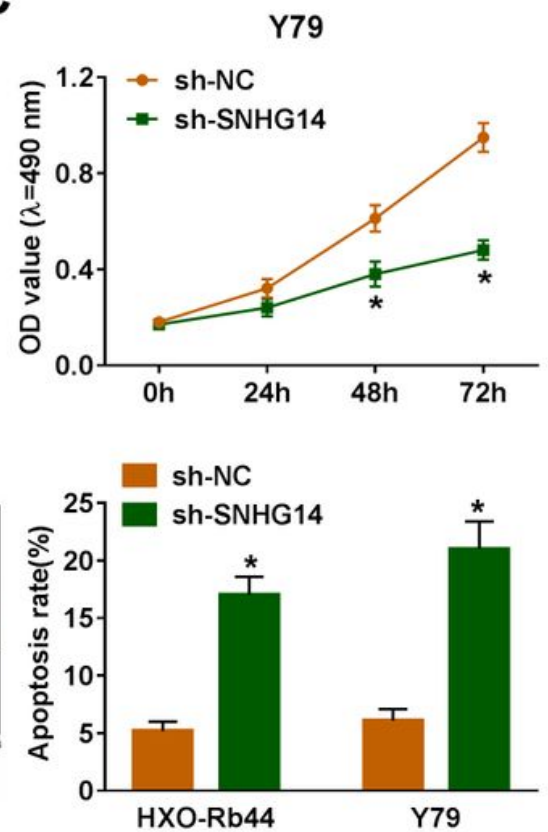

Y79

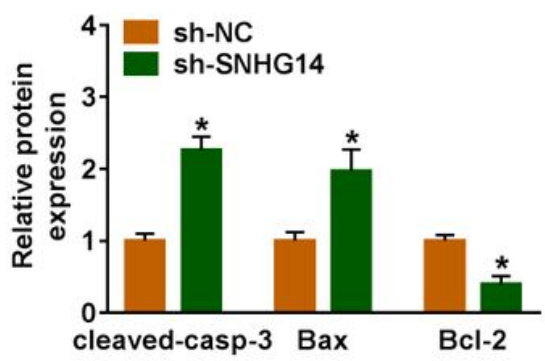

\section{Figure 2}

The role of SNHG14 in retinoblastoma cells. (A) Transfection efficiency of sh-SNHG14 was confirmed by qRTPCR. (B, C) Cell proliferation was detected by CCK-8 assay. (D) Cell apoptosis rate was performed via Flow cytometry. (E) Levels of cleaved-casp-3, Bcl-2 and Bax were detected using western blot. ${ }^{*} \mathrm{P}<0.05$. 
A
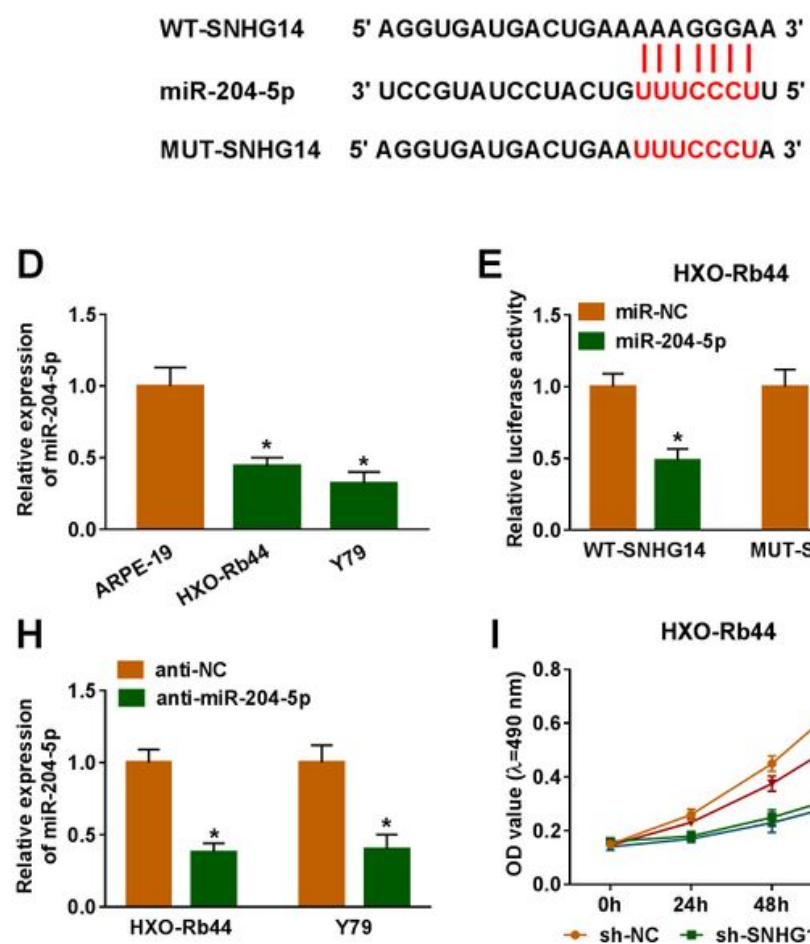

L

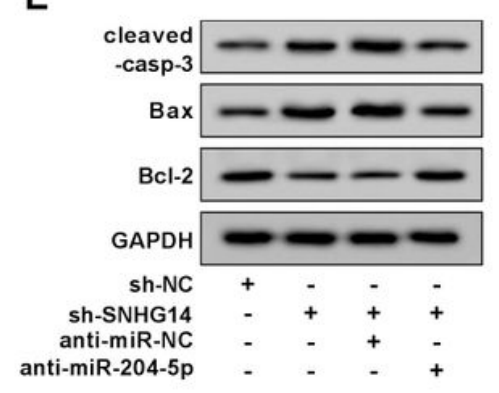

E
B
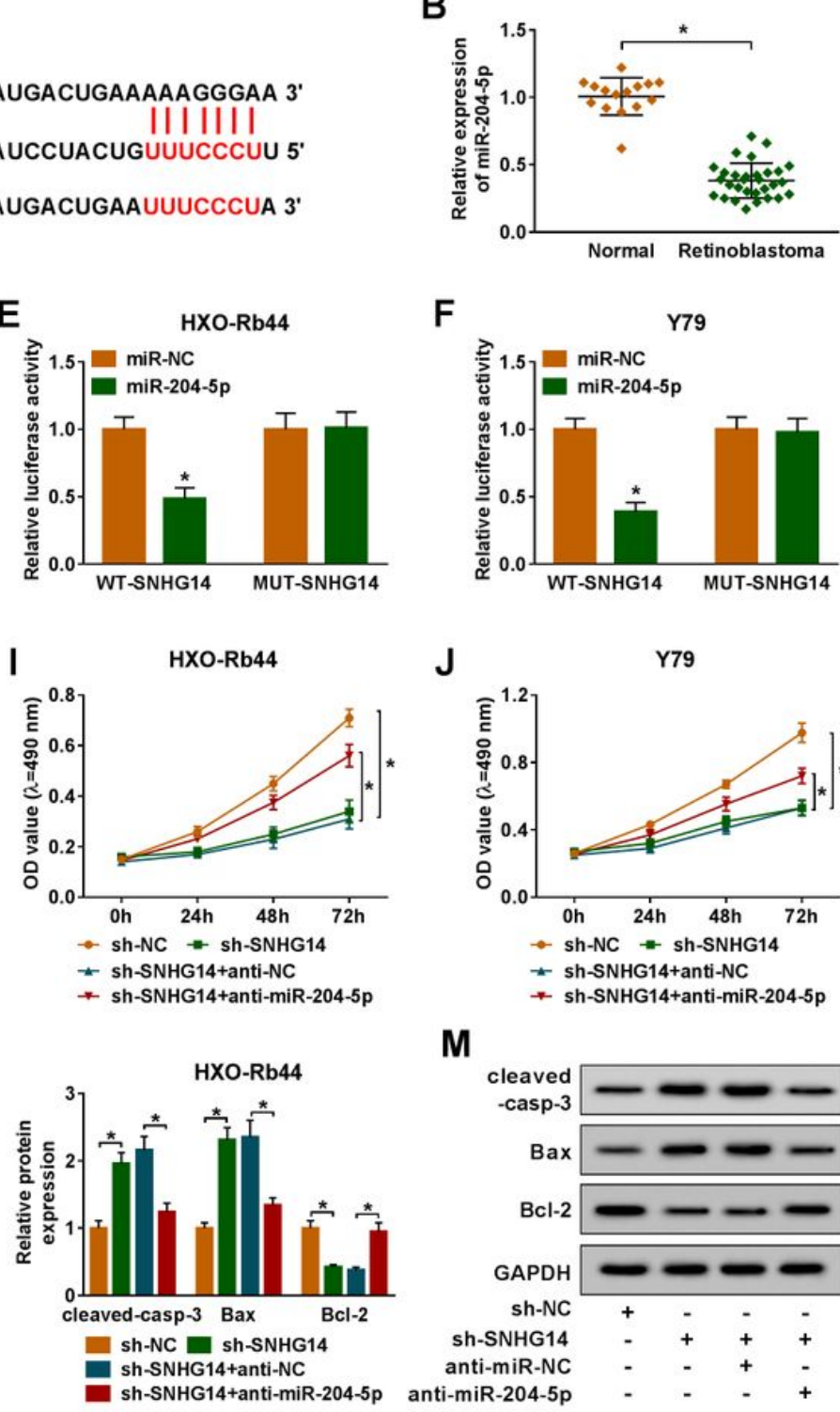

F

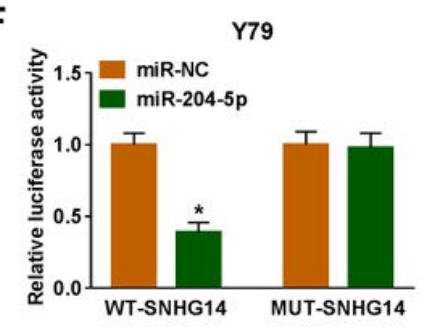

J

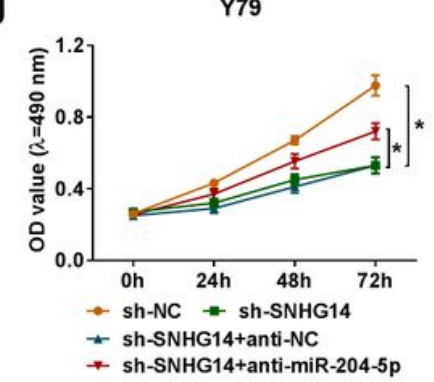

M

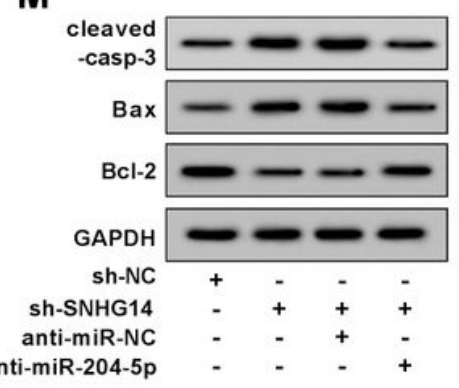

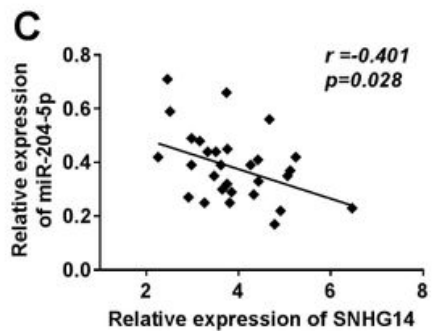

G

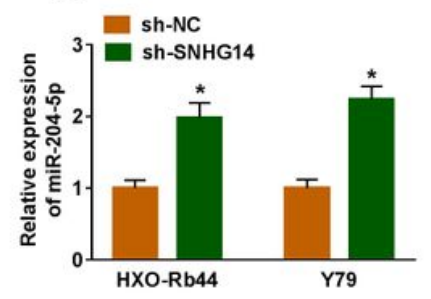

K
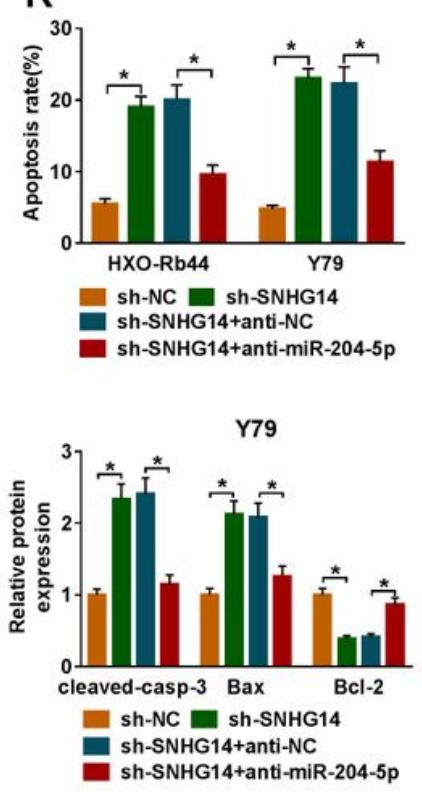

Figure 3

SNHG14 targeted miR-204-5p to promote retinoblastoma progression. (A) The putative binding sites of SNHG14 and miR-204-5p. (B) Levels detection of SNHG14 in retinoblastoma tissues and normal retina samples with qRT-PCR. (C)The relationship between miR-204-5p and SNHG14 in retinoblastoma tissues was measured. (D) Levels detection of SNHG14 in human retinoblastoma cell lines (HXO-RB44, and Y79) and normal cell line ARPE-19 using qRT-PCR. (E, F) Luciferase activity was examined in HXO-Rb44 and Y79 cells co-transfected with WT-SNHG14 or MUT-SNHG14 and miR-NC or miR-204-5p. (G)The expression of miR-204$5 p$ was measured in HXO-Rb44 and $Y 79$ cells transfected with sh-SNHG14 or sh-NC by qRT-PCR. $(\mathrm{H})$ The level of miR-204-5p in HXO-Rb44 and Y79 cells transfected with anit-NC, or ant-miR-204-5p using qRT-PCR. HXORb44 and Y79 cells were transfected with sh-NC, sh-SNHG14, sh-SNHG14 + anti-NC or sh-SNHG14 + anti-miR204-5p. After transfection, $(\mathrm{I}, \mathrm{J})$ cell proliferation was detected using acck-8 assay; $(\mathrm{K})$ flow cytometry analysis of cell apoptosis rates; $(L, M)$ western blot was used to examine the levels of cleaved-casp-3, Bcl-2 and Bax. * $P<0.05$. 
A

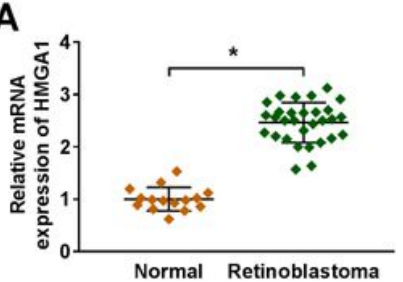

D

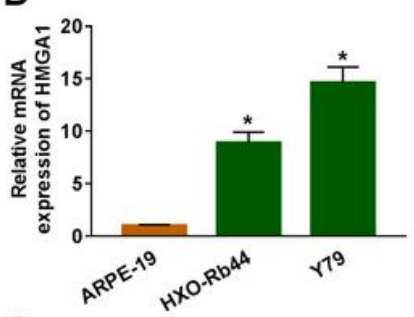

G

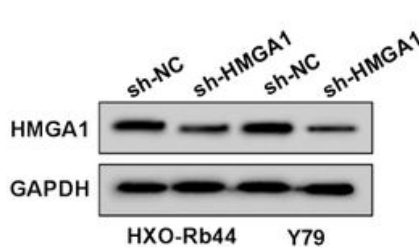

J

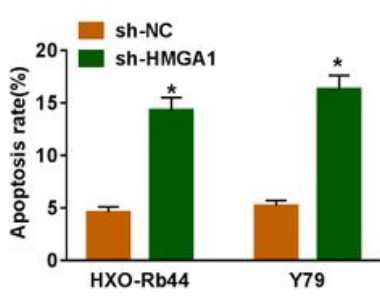

B

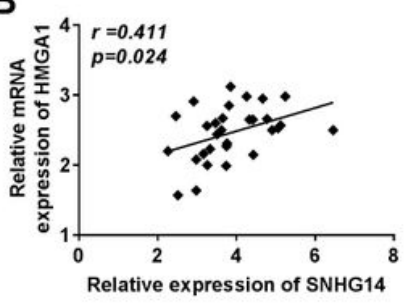

E
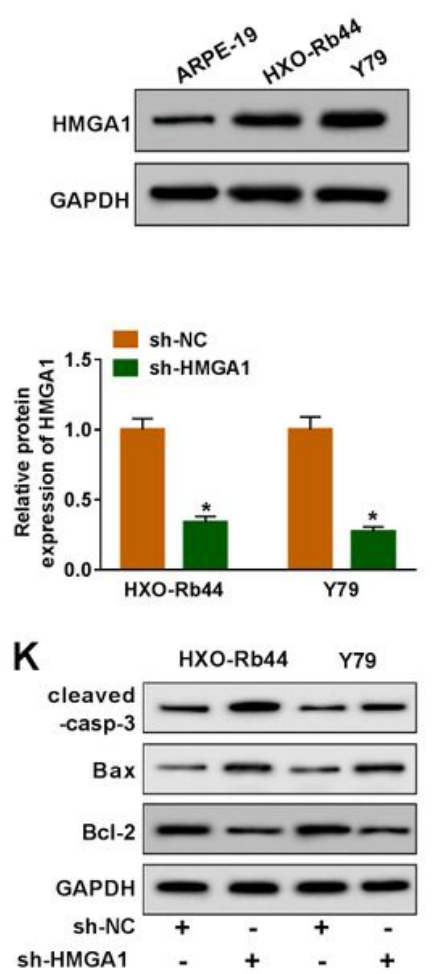

C
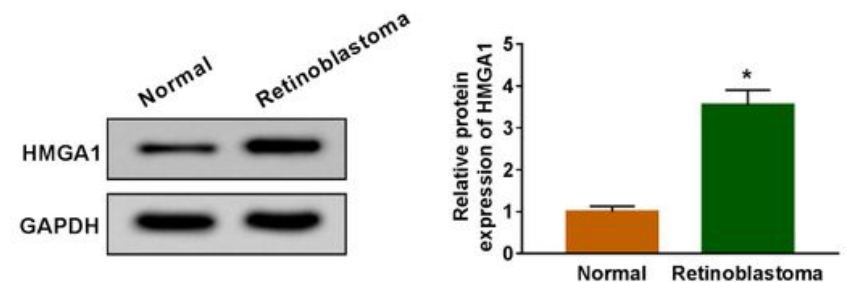

$\mathbf{F}$

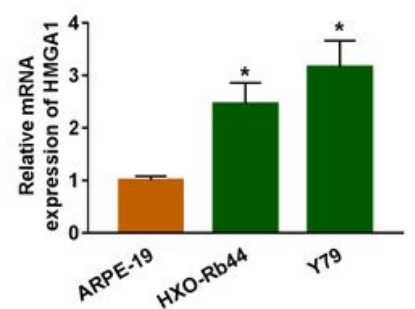

HXO-Rb44

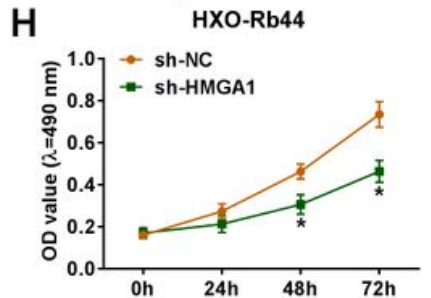

I
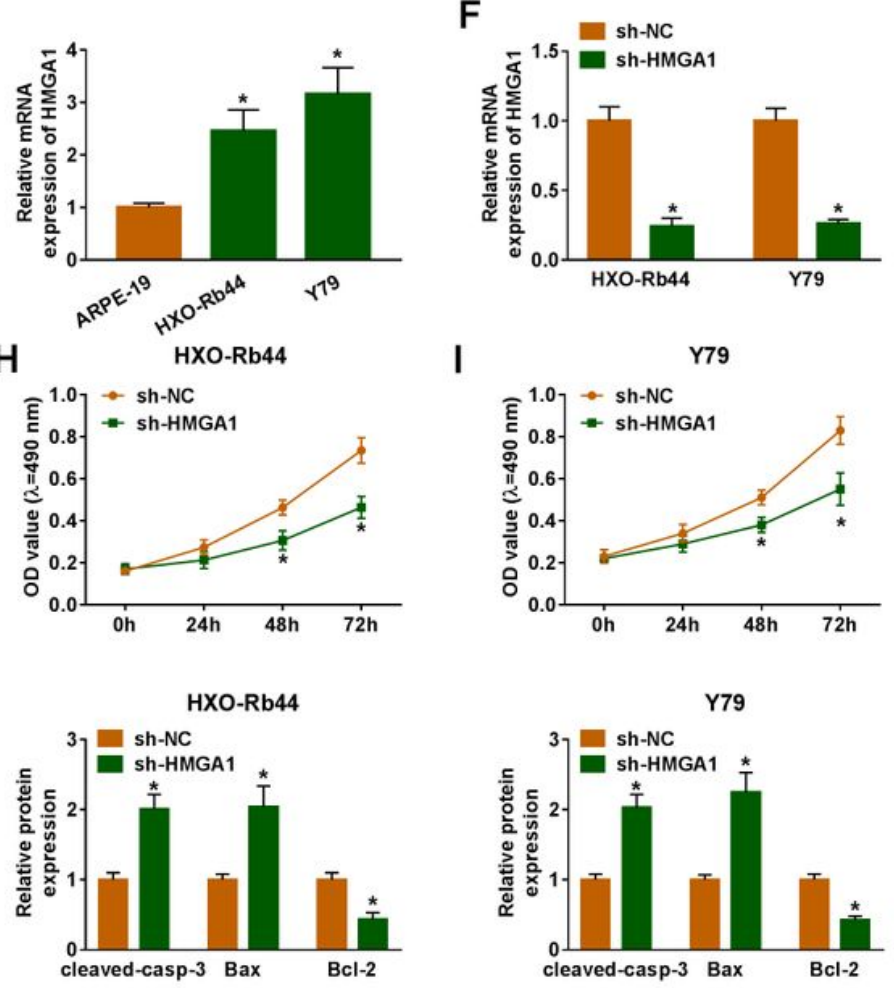

\section{Figure 4}

The expression and effects of HMGA1 on retinoblastoma. (A, C) Levels detection of HMGA1 mRNA (A) and protein $(C)$ in retinoblastoma tissues and normal retina samples with qRT-PCR and western blot. (B) The relationship between miR-204-5p and HMGA1 in retinoblastoma tissues was analyzed. (D, E) Levels detection of HMGA1 mRNA (D) and protein (E) in human retinoblastoma cell lines (HXO-RB44, and Y79) and normal cell line ARPE-19 using qRT-PCR and western blot. (F, G) Transfection efficiency of sh-HMGA1 or sh-NC was confirmed by qRT-PCR (F) or western blot (G) in HXO-Rb44 and Y79 cells. (H, I) CCK-8 analysis of cell proliferation. (J) Cell apoptosis was detected using flow cytometry. (K) Levels of cleaved-casp-3, Bcl-2 and Bax were measured using western blot. ${ }^{*} \mathrm{P}<0.05$. 
A

HMGA1 3'UTR-WT 5' GGUGUUCCAUGAGGAAAAGGGAA 3' miR-204-5p 3' UCCGUAUCCUACUGUUUCCCUU 5'

HMGA1 3'UTR-MUT 5' GGUGUUCCAUGAGGAUUUCCCUA 3'

D

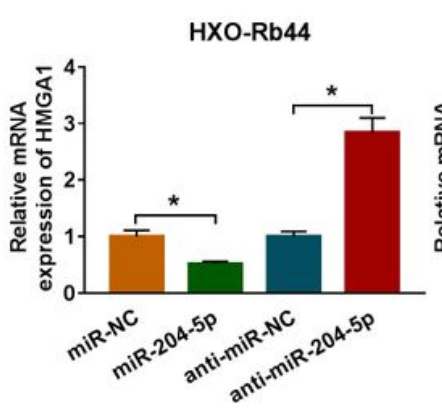

B
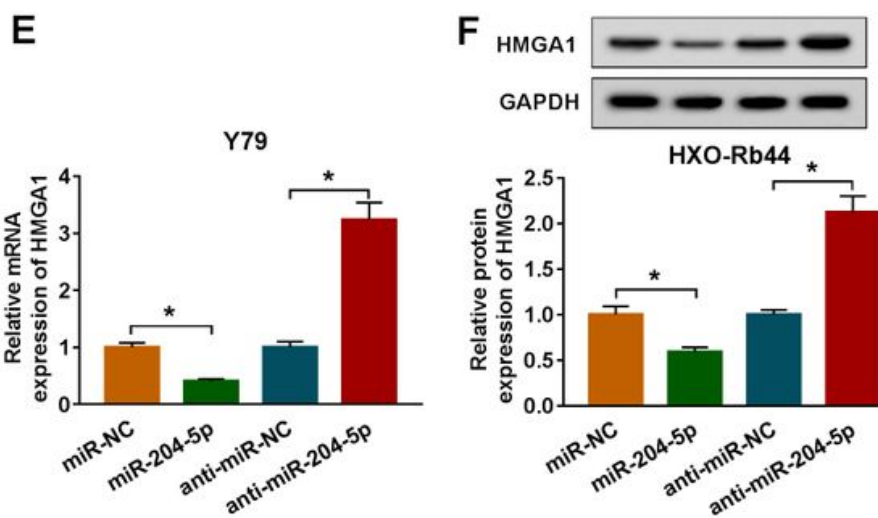

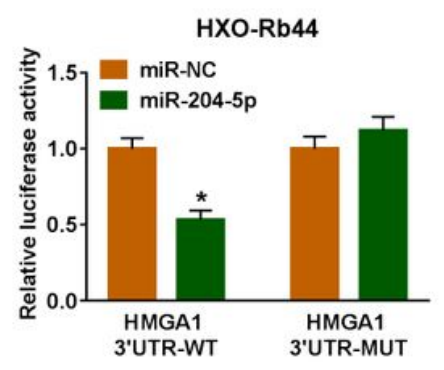

HXO-Rb44

C
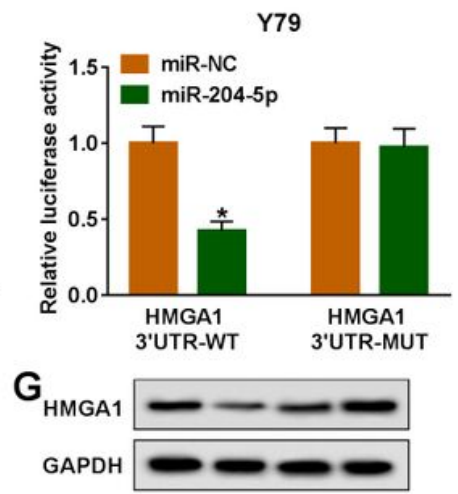

Y79
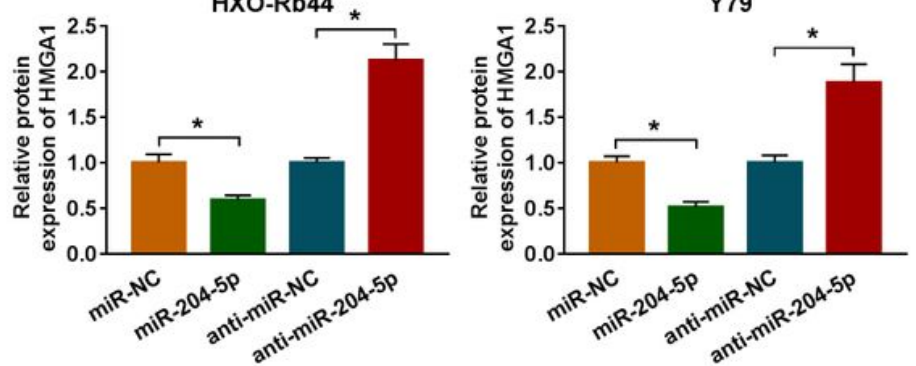

Figure 5

HMGA1 is a target of miR-204-5p. (A) The putative binding sites of HMGA1 and miR-204-5p. (B, C) Luciferase activity was examined in HXO-Rb44 (B) and Y79 (C) cells co-transfected with HMGA1 3'UTR-WT or HMGA1 3'UTR-MUT and miR-NC or miR-204-5p. (D-G) The mRNA and protein expression of HMGA1 in HXO-Rb44 and Y79 cells transfected with miR-NC, miR-204-5p, anti-miR-NC or anti-miR-204-5p was detected by qRT-PCR or western blot, respectively. ${ }^{\star} \mathrm{P}<0.05$. 
A

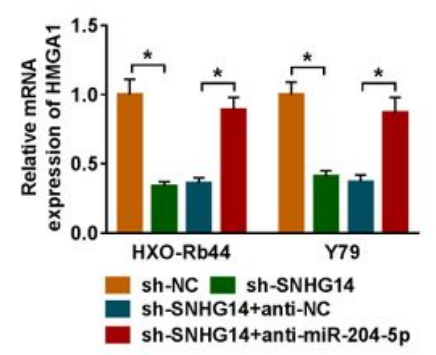

C

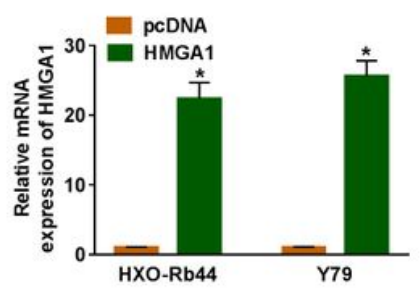

$\mathbf{F}$
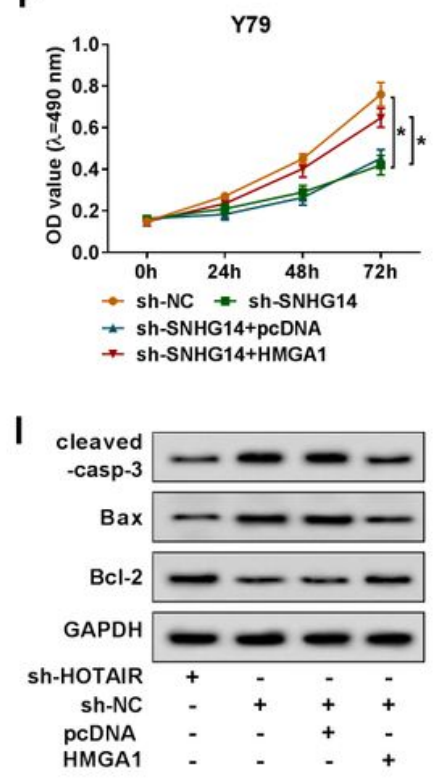

B
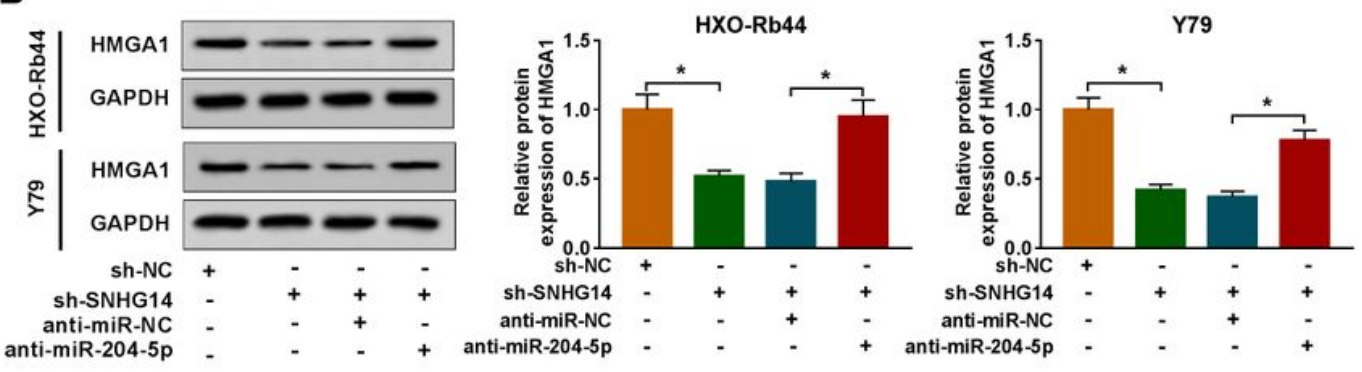

D

$\mathrm{E}$
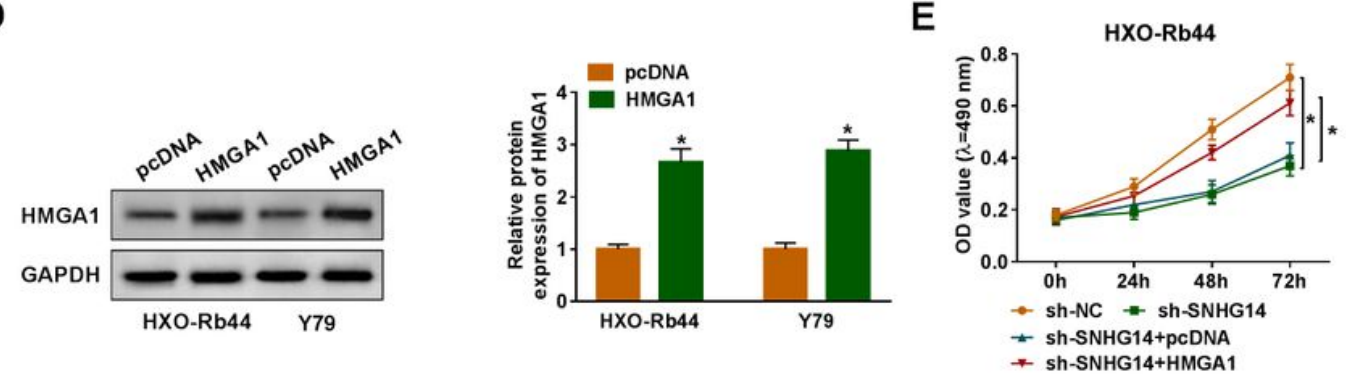

G

$\mathrm{H}$
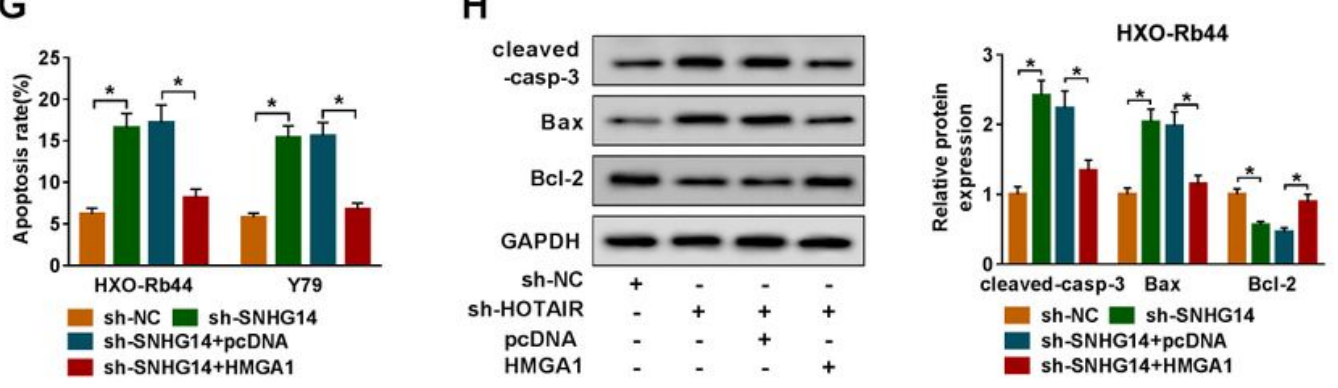

\section{Figure 6}

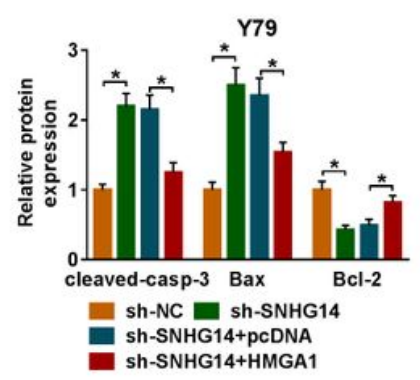

SNHG14 inhibits proliferation and induces apoptosis via regulating miR-204-5p/HMGA1 axis in retinoblastoma cells. HXO-Rb44 and Y79 cells were transfected with sh-NC, sh-SNHG14, sh-SNHG14 + anti-NC or sh-SNHG14 + anti-miR-204-5p. After transfection, (A, B) the expression of HMGA1 in HXO-Rb44 and Y79 cells was detected by qRT-PCR or western blot, respectively. (C, D) Levels of HMGA1 in HXO-Rb44 and Y79 cells transfected with pcDNA or HMGA1 were detected using qRT-PCR or western blot, respectively. HXO-Rb44 and $Y 79$ cells were transfected with sh-NC, sh-SNHG14, sh-SNHG14 + pcDNA, or sh-SNHG14 + HMGA1. After transfection, (E, F) CCK-8 analysis of cell proliferation; $(\mathrm{G})$ cell apoptosis analysis using flow cytometry. $(\mathrm{H}, \mathrm{I})$ Levels of cleaved-casp-3, Bcl-2 and Bax were measured using western blot. ${ }^{\star} \mathrm{P}<0.05$. 
A

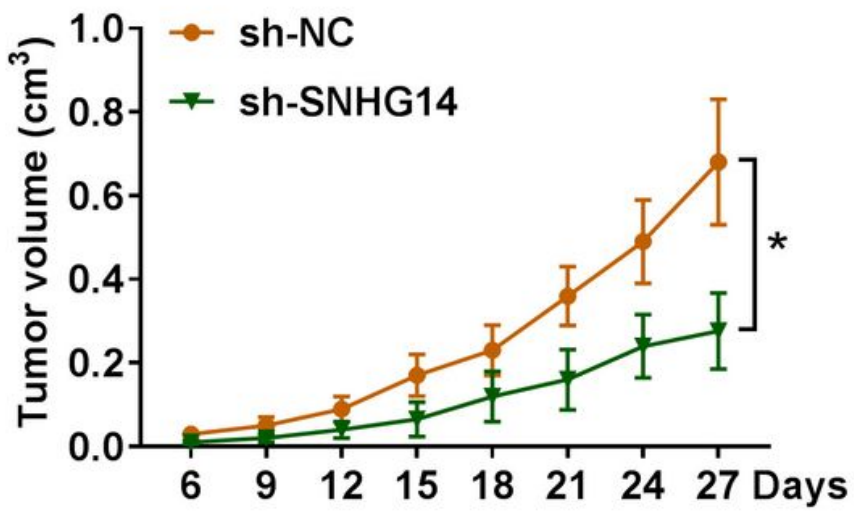

C

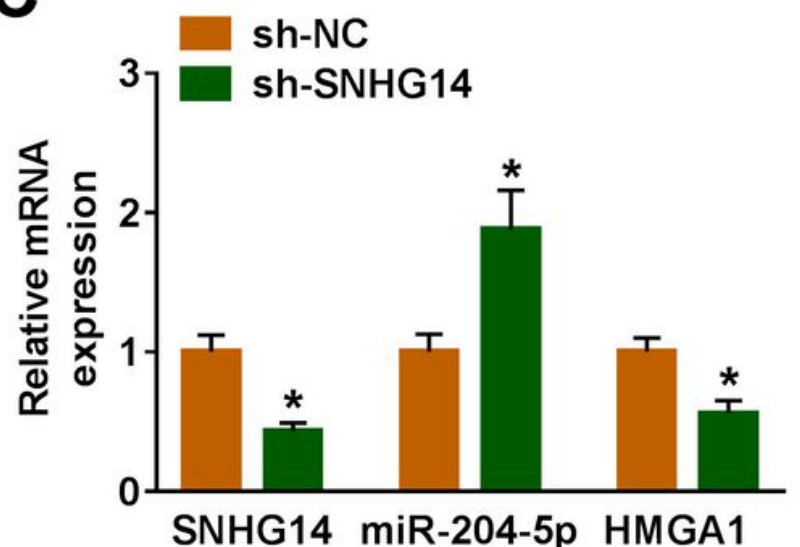

B
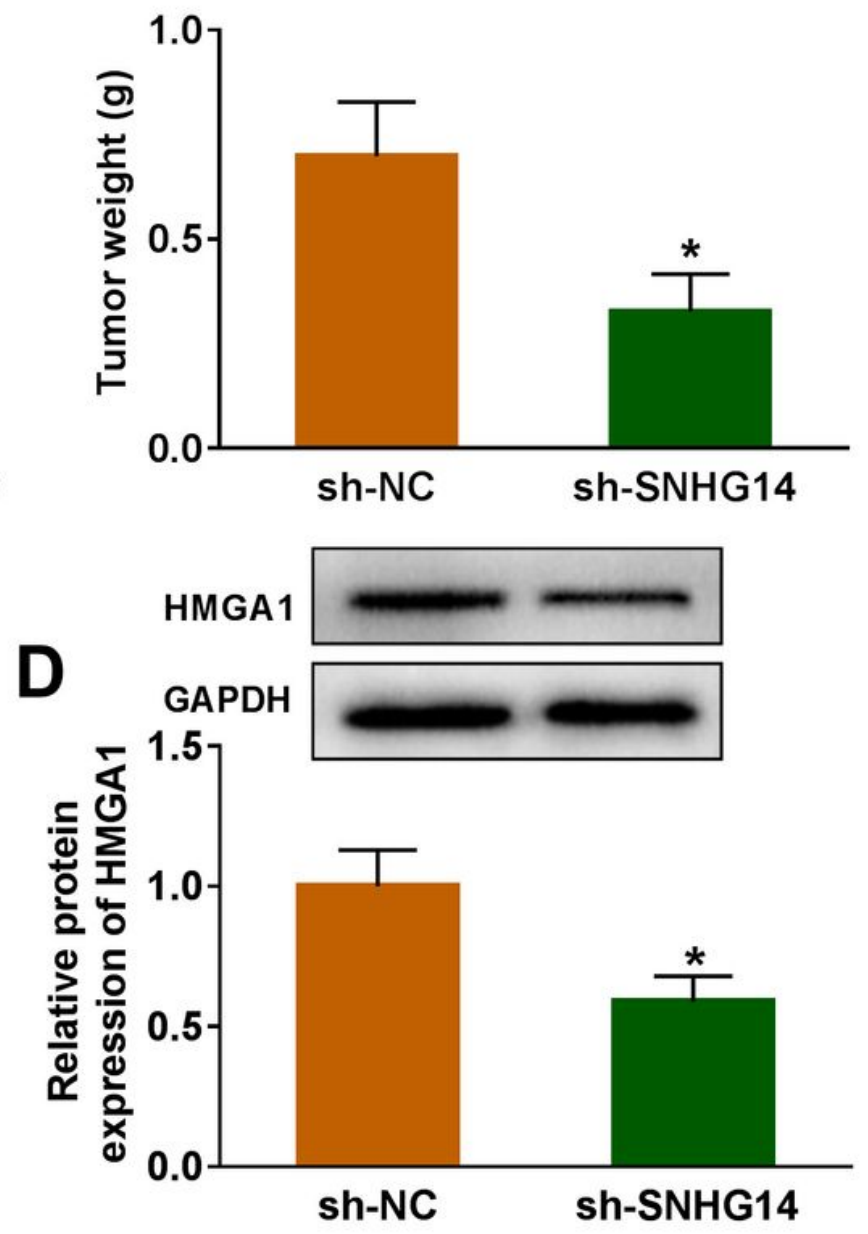

Figure 7

SNHG14 knockdown inhibits retinoblastoma tumor growth in vivo. (A, B) Tumor volume and weight analysis of tumor masses from xenografts with SNHG14 down-regulated Y79 cells and negative control Y79 cells. (C) qRT-PCR analysis of SNHG14, miR-204-5p and HMGA1 in tumor mass from xenografts. (D) Western blot analysis of HMGA1 level in tumor mass from xenografts. ${ }^{*} \mathrm{P}<0.05$. 